\title{
A New Skin and Frontal Face Detector by selecting Optimized Color Spaces
}

\author{
B. Somayeh Mousavi \\ Young Researcher Club, \\ Islamic Azad University, \\ Zahedan Branch, Zahedan, \\ Iran.
}

\author{
Amin Shahraki \\ Moghadam \\ Computer Department, Islamic \\ Azad University, Zahedan \\ Branch, Zahedan, Iran.
}

\author{
Mohammad Mojtaba \\ Keikhayfarzaneh \\ Department of Psychology, \\ Young Researcher Club, \\ Islamic Azad University, \\ Zahedan Branch, Zahedan, \\ Iran
}

\begin{abstract}
In this paper a new method to detect faces in colour images, is proposed which has wide range of application in HumanComputer interaction, face processing and illness diagnosis (specially mental disease). Skin colour, lip position and face shape information are the key parameters for developing a fussy rule based classifier to extract face candidate from an image. In first step, skin regions are identified in HSI colour space, using fuzzy system, applying distance of each pixel colour to skin colour cluster as input and produce a skin-likelihood image in output. Next step is searching lip area in the region owning the most likelihood of belonging to skin. This stage is implemented in normalized RGB colour space by fuzzy system its input is $\mathrm{r}-\mathrm{g}$ value. To extract face regions, fuzzy rule based system is used, applying face and lip position, lip area data and face shape to decide which skin region is human face. With this algorithm, $98.75 \%, 86.6 \%$ detection rates are achieved, respectively for frontal and near frontal. Revising system for detecting profile and rotating faces associated with exactly determine lip area, is the next goal.
\end{abstract}

\section{KEY WORDS}

face detection, lip detection, colour space, fuzzy rule based system, mental illness diagnosis.

\section{INTRODUCTION}

Face detection, defined as detection and localization of human faces in images, has been challenging topic in human computer interaction (HCI) researches. Human face detection application is observable mainly in video surveillance, face recognition, mental illness diagnosis by face processing and image databases management.

The problem associated with face detection can be attributed to the following factors [1]:

- Pose: Due to relative camera-face pose, it might some facial features, such as: an eye or the nose, become partially or wholly occluded.

- $\quad$ Presence or absence of structural components: Facial feature such as beards, moustaches and glasses may or may not present; moreover, it cause some components including shape, colour and size be variable.

- $\quad$ Facial expression: Facial expression affect directly on appearance of faces.

- Occlusion: Some faces in an image, may partially occlude other faces.

- Image orientation: Face images directly vary for different rotation about the camera's optical axis.
- Image condition: some factors such as lighting and camera characteristics can affect face appearance.

In general, single image detection methods can be classified in to four categories [1]:

1. Knowledge-based methods.

2. Feature invariant approaches.

3. Template matching methods.

4. Appearance-based methods.

Among methods which lie within the second category, using skin colour is one of the most useful approaches in many applications, from face detection to hand tracking. It has been proved that the differ of skin colour in different peoples is more due to intensity [1].

Most of research efforts on skin detection have focused on visible spectrum imaging. However; skin colour detection in visible spectrum is sensitive to various factors such as [2]:

- Illumination: changing light source distribution and illumination level (indoor, outdoor, highlights, shadows, non-white lights) cause changing in the colour of the skin in the image. The illumination variation is the most important problem among current skin detection systems.

Camera characteristics: The skin colour distribution for the same person differs from one camera to another, even under the same illumination.

- $\quad$ Ethnicity: Skin colour varies from person to person belonging to different ethnic groups and also from persons across different regions..

- Individual characteristics: Age, sex and body parts are examples of individual characteristics affecting the skin colour appearance.

- Other factors: Various factors such as subject appearances (make up, hairstyle and glasses), background colours, shadows and motion also influence skin colour appearance.

Although many of the problems, mentioned above, can be overcome by using non-visual spectrum such as infrared (IR) and spectral imaging, the expensive equipment necessary for these methods combined with tedious setup procedures have limited their use to specific application areas such as biomedical applications. In continue, visual spectrum-based skin detection techniques that are applicable for 2D images or single frames of video, are considered.

When building a system, utilizing skin colour as a feature for face detection, three main questions would be arisen. First, what colour space to choose. Second, how the skin colour distribution should be modelled and third, what will be the way of processing of colour segmentation results for face detection?

The choice of colour space is considered as the primary step in skin-colour classification. The RGB colour space is the 
default colour space for most available image formats. Any other colour spaces could be obtained from a linear or nonlinear transformation from RGB.

Several colour spaces have been proposed for skin detection. The most widely used colour spaces are classified as follow [2]:

1. Basic colour spaces (RGB [3], normalised RGB [4], CIE$\mathrm{XYZ):} \mathrm{RGB} \mathrm{correspond} \mathrm{to} \mathrm{the} \mathrm{three} \mathrm{primary} \mathrm{colours:} \mathrm{red,}$ green and blue. To reduce the dependence on lighting, the RGB colour components are normalised so that sum of the normalised components are unity $(\mathrm{r}+\mathrm{g}+\mathrm{b}=1)$; as the result, the third component does not hold any significant data and is normally dropped. The CIE (Commission International de l'Eclairage) system describes colour as a luminance component $\mathrm{Y}$, and two additional components $\mathrm{X}$ and $\mathrm{Z}$. CIE-XYZ values were constructed from psychophysical experiments and correspond to the colour matching characteristic of human visual system.

2. Perceptual colour spaces (HSI, HSV [5], HSL, TSL): The perceptual features of colour such as hue $(\mathrm{H})$, saturation $(\mathrm{S})$ and intensity (I) can not be described directly by RGB so, many non-linear transformation are proposed to map RGB on to perceptual features.

3. Orthogonal colour spaces (YCbCr [6], YIQ, YUV, YEs): The orthogonal colour spaces represent the colour with statistically independent components.

4. Perceptually uniform colour spaces (IE-Lab and CIE-Luv): In these colour spaces, the computation of the luminance (L) and the chroma (ab or uv) is obtained through a nonlinear mapping of the XYZ coordinates.

5. Other colour spaces: These colour spaces are created by mixture of other spaces or using colour ratio.

After choosing suitable colour space, the decision rule to discriminate between skin and non-skin pixels should be built. To this aim, four group of skin modeling methods are utilized [7]:

1. Explicitly defined skin region

2. Nonparametric skin distribution modeling.

3. Parametric skin distribution.

4. Dynamic skin distribution models.

Many researches are implemented to compare different methods in different colour spaces [8-12].

In this paper, the new approach is developed using skin and lip colour analysis and fuzzy theory, to detect human faces in colour images. Beside colour information, other properties of lip and face and face shape are utilized to verify the face detection. The proposed method is described in the next sections, by details.

\section{FUZZY RULE BASE SYSTEM}

A rule-based system consist of a set of if-then rules, a set of facts and an interpreter controlling the application of the rules, given the facts. The building blocks of the rule-based system can be crisp or fuzzy.

Among all the techniques developed using fuzzy sets, fuzzy if-then rules are by far the most visible due to their wide range of successful applications. Fuzzy if-then rules have been applied to many disciplines such as control systems, decision making and pattern recognition.

Fuzzy rule based systems contain a rule base, a decision making unit, and finally a defuzzifacation interface. The function of each block is as follow [13]:

- A rule base containing a number of fuzzy if-then rules.

- A database which defines the membership functions of the fuzzy sets used in the fuzzy rules.

- A decision-making unit which performs the inference operation on the rules.
- A fuzzification interface which transforms the crisp input in to degrees of match with linguistic values.

- A defuzzification interface which transform the fuzzy results of the interface in to crisp output.

The most important two types of fuzzy inference methods are Mamdani and Sugeno or Takagi-Sugeno-Kung methods. Mamdani type is the most commonly seen inference method which is used in this paper, so it will be described in more details. This method consist of the following linguistic rules that describe a mapping from $U_{1} \mathrm{x} U_{2} \mathrm{x} \ldots \mathrm{x} U_{r}$ to $W$ [14]

$R_{i}$ : IF $x_{1}$ is $A_{i l}$ and...and $x_{r}$ is $A_{i r}$ THEN $y$ is $C_{i}$, where $x_{j}$ $(j=1,2, \ldots, r)$ are the input variables, $y$ is the output variable, and $A_{i j}$ and $C_{\mathrm{i}}$ are fuzzy sets for $x_{j}$ and $y$ respectively; Given input of the form: $x_{1}$ is $A_{1}^{\prime}, x_{2}$ is $A_{2}^{\prime}, \ldots, x_{r}$ is $A_{r}^{\prime}$. Where $A_{1}^{\prime}, A_{2}^{\prime}, \ldots, A_{r}^{\prime}$ are fuzzy subsets of $U_{l}, U_{2}, \ldots, U_{r}$, the contribution of rule $R_{i}$ to Mamdani model's output is a fuzzy set whose membership function is

$$
\mu_{C^{\prime}}(y)=\left(\alpha_{i 1} \wedge \alpha_{i 2} \wedge \ldots \wedge \alpha_{i n}\right) \wedge \mu_{C_{i}}(y) .
$$

Where $\alpha_{i}$ is the matching degree of rule $R_{i}$, and where $\alpha_{i j}$ is the matching degree between $x_{j}$ and $R_{i}$ 's condition on $x_{j}$..

$\alpha_{i j}=\sup \left(\mu_{A_{j}^{\prime}}\left(x_{j}\right) \wedge \mu_{A_{i j}}\left(x_{j}\right)\right)$.

and $\wedge$ denotes the "min" operator.

The final output of the model is the aggregation of outputs from all rules using the max operator;

$$
\mu_{C}(y)=\max \left\{\mu_{C_{1}^{\prime}}(y), \mu_{C_{2}^{\prime}}(y), \ldots, \mu_{C_{L}^{\prime}}(y)\right\} \text {. }
$$

The output $C$ is a fuzzy set. This fuzzy output can be deffuzzified in to a crisp output using one of the deffuzzification techniques.

\section{3. .PROPOSED METHOD}

\subsection{COLOUR SPACE SELECTION}

The transformation of RGB to perceptual colour spaces is invariant to high intensity at white lights, ambient light and surface orientations relative to the light source; consequently, they can be a so suitable choice for skin detection methods.

By following non-linear equation, RGB colour space is transform to HSI colour space, which has the advantage that intensity component is separated from chrominance components:

$$
\begin{gathered}
H=\arccos \frac{\frac{1}{2}((R-G)+(R-B))}{\sqrt{\left((R-G)^{2}+(R-B)(G-B)\right)}} . \\
S=1-3 \frac{\operatorname{Min}(R, G, B)}{R+G+B} . \\
I=\frac{1}{3}(R+G+B) .
\end{gathered}
$$

$2 \mathrm{D}$ projection of $3 \mathrm{D}$ colour distribution can not adapts itself to lighting variation; furthermore, simply discarding luminance information affects the model's accuracy [12].

\subsection{SKIN AND LIP COLOUR SEGMENTATION}

After transform input image to selected colour space, next step is finding skin pixels. To this goal, the Euclidean distance of the colour of each pixel to skin colour sub-space, is used as 
an input for a fuzzy system. Fuzzy system is a mamdani type, 1input, 1-output system. To achieve crisp output centroid method is chosen, which is the most widely used one among seven defuzzification approaches [13]. The membership functions (MFs) of input and output is depicted in "Fig. 1".

The fuzzy linguistic terms of input (d) are: \{Face, Rather Face, Low Probability Face, Not Face\}. Similarly, the terms set of output (p) is: \{Face, Rather Face, Low Probability Face, Not Face\}.

The result of apply such a system is the skin-likelihood image; that is, the gray-scale image whose gray values represent the likelihood of the pixel belonging to skin. The pixels with $89 \%$ likelihood or more are regarded as skin pixels. The binary image is formed by setting skin pixels to 1 and all other pixels to 0 . After this, morphological processing, consist of filling holes and opening followed by closing [15], is accomplished to acquire separate and connected regions. Each region is applied as an input for next step

Lip colour segmentation part, searches whole skin regions to find lip pixels, one by one. Finding lip pixels system describes as follow:

The target region is transformed to normalised RGB colour space. This colour space is achieved using "(5)".

$$
\begin{aligned}
& r(x, y)=\frac{R(x, y)}{\operatorname{Max}(R, G, B)} \\
& g(x, y)=\frac{G(x, y)}{\operatorname{Max}(R, G, B)} \\
& b(x, y)=\frac{B(x, y)}{\operatorname{Max}(R, G, B)}
\end{aligned}
$$

This colour space has been shown best result in finding lip cases [4]. To reduce false positive in lip detection, lip pixels are searched just in the lower half region. With this technique false positive, causing by redness of cheeks is ignored.

In normalised RGB colour space and in desired region, the $r$ $\mathrm{g}$ values are utilized as fuzzy system inputs. The properties of this system are same as the skin colour segmentation system, except the membership functions shapes. The MFs are shown in "Fig. 2".

The input ( $\mathrm{r}-\mathrm{g})$ is recognized by linguistic terms: $\{\mathrm{Lip}$, Rather Lip, Not Lip\}; likewise, for the output (l) linguistic terms are: $\{$ Lip, Rather Lip, Not Lip $\}$. Similar to skin detection system, the result is lip-likelihood image. $84 \%$ likelihood is chosen as the threshold to make binary image of lip regions.

Fill the holes and erosion plus opening by reconstruction [15], are the morphological processing done at the end of this stage.

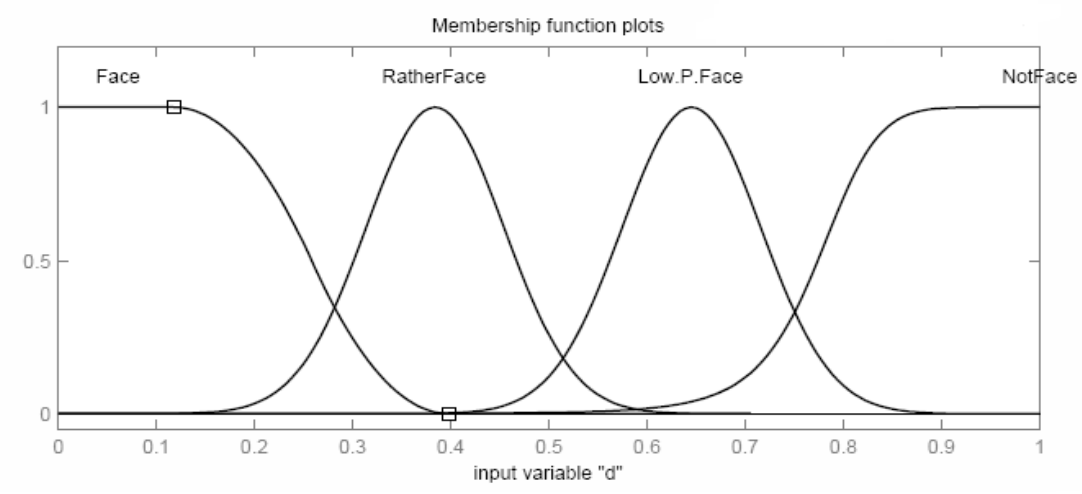

(a)

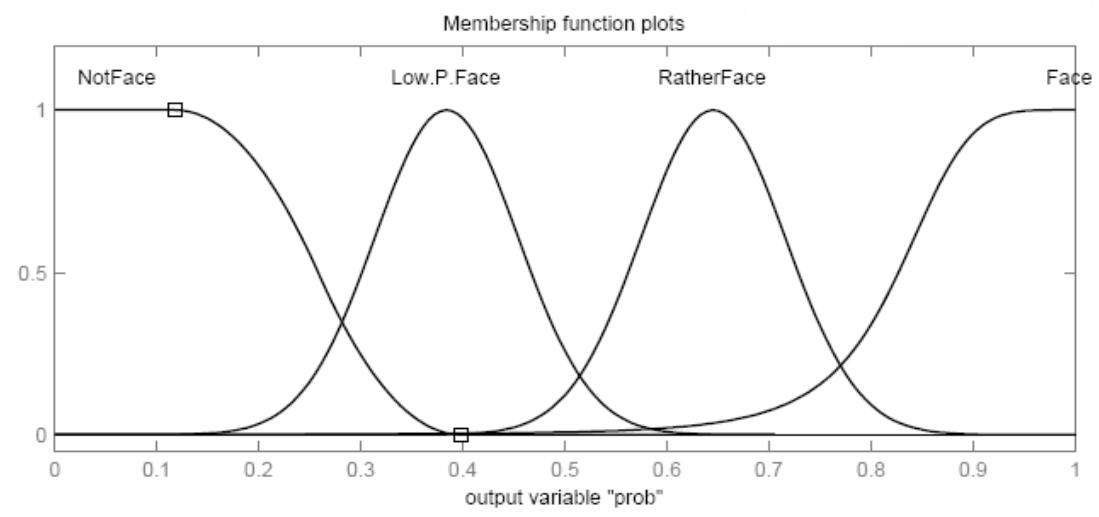

(b)

Figure 1. (a) input and (b) output MFs for skin colour segmentation fuzzy classifier 


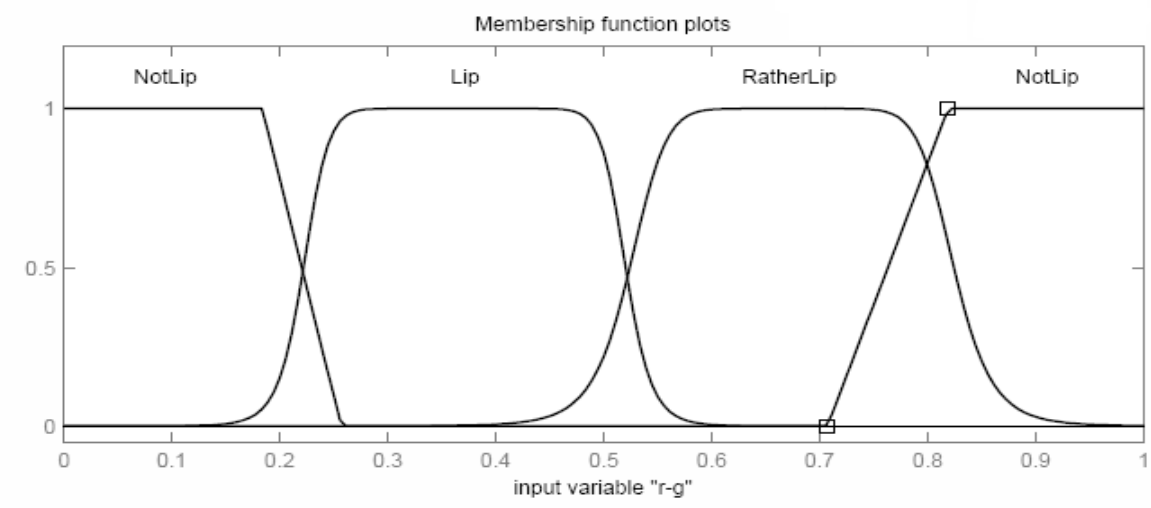

(a)

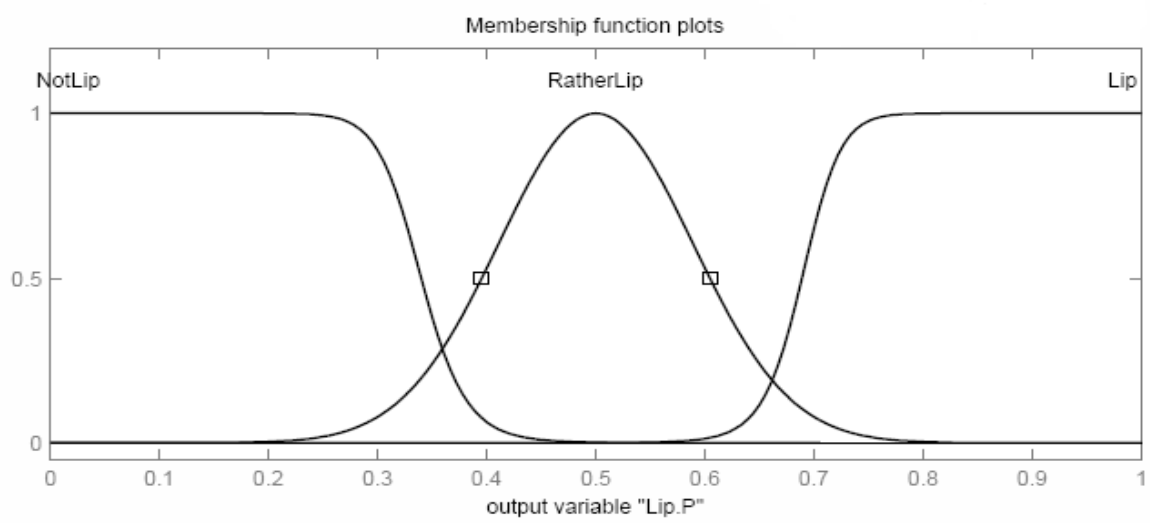

(b)

Figure 2. (a)input and (b) output MFs for lip colour segmentation fuzzy classifier

\subsection{DETECTING FACE ALGORITHM}

In final step, the face blobs must be selected among skin regions. Deciding system is a fuzzy system with three inputs and one output. The first input (A) is the ratio of lip area to face area. The second input (B) is the tangent of angle between maximum length line of face area and minimum length line of lip area. These two lines should be parallel, approximately. Angles less than $30^{\circ}$ are acceptable. The third input (C) reveals the similarity of region shape to an ellipse. This ellipse estimates the face shape. Output (Y) expresses likelihood of the region being face. Region with more than $80 \%$ likelihood is introduced as a face.

The inputs and output MFs and an example of composition of rules, are illustrated in "Fig. 3" and "Fig. 4". In this example the output $\mathrm{Y}=0.832$ is achieved for the inputs $\mathrm{A}=0.1, \mathrm{~B}=0.5$ and $\mathrm{C}=0.8$.

\section{OBTAINED RESULTS}

To test the proposed approach, 100 images contain frontal, near frontal and profile faces, are selected. These images are gathered among Bao and Caltech [16] image datasets. The image sizes are between $125^{*} 93$ to $296^{*} 448$ pixels.

The detection results are summarized in table I. Because of method dependency to lip detection, it is much more applicable for frontal faces. However, the method shows nearly acceptable result for near frontal faces. As is predictable, the algorithm fails in detection of profile faces in most cases and just $20 \%$ detection rate is achieved..

"Fig. 5" shows instance of applying proposed algorithm to a picture. The step-by-step results of different stages, described in previous sections, are observable. Whole described stages are converted to computer program using MATLAB software.

TABle 1. ExPERIMENTAL Results (DR: Detection RATE)

\begin{tabular}{|c|c|c|}
\hline Head Pose & Frontal & Near-Frontal \\
\hline $\begin{array}{c}\text { Number of } \\
\text { Images }\end{array}$ & 80 & 15 \\
\hline DR(\%) & 98.75 & 86.6 \\
\hline
\end{tabular}




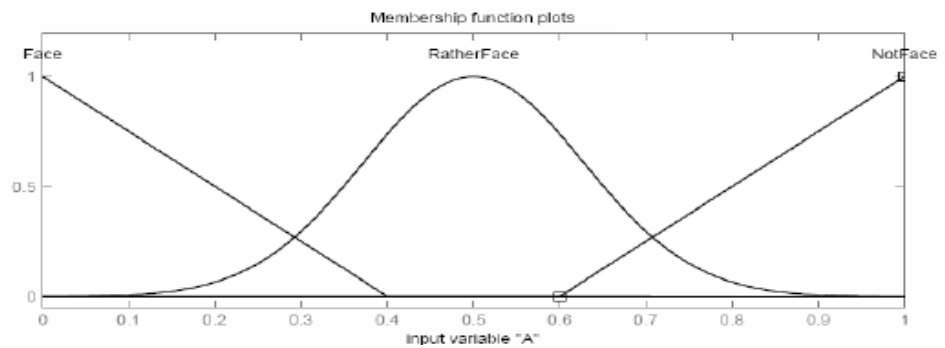

(a)

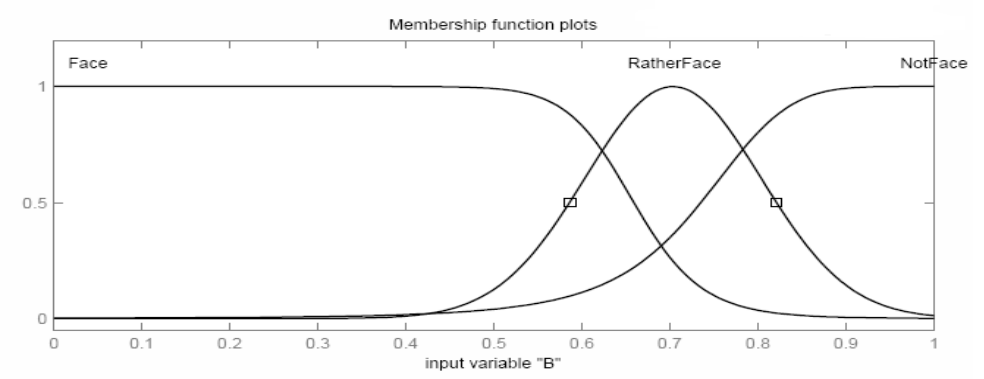

(b)

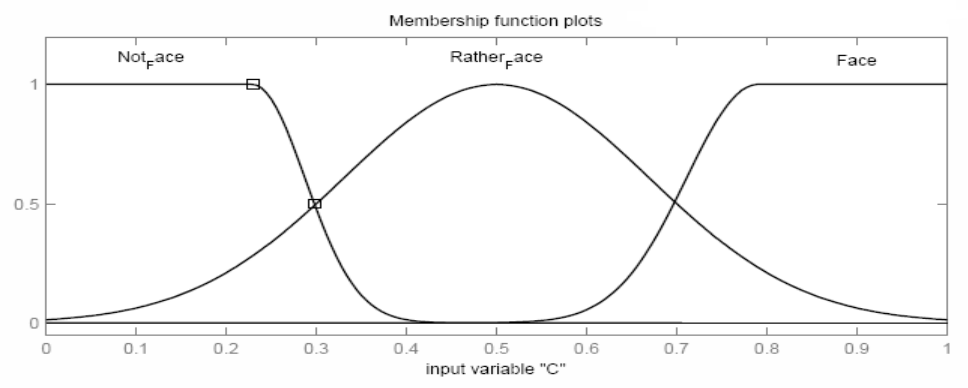

(c)

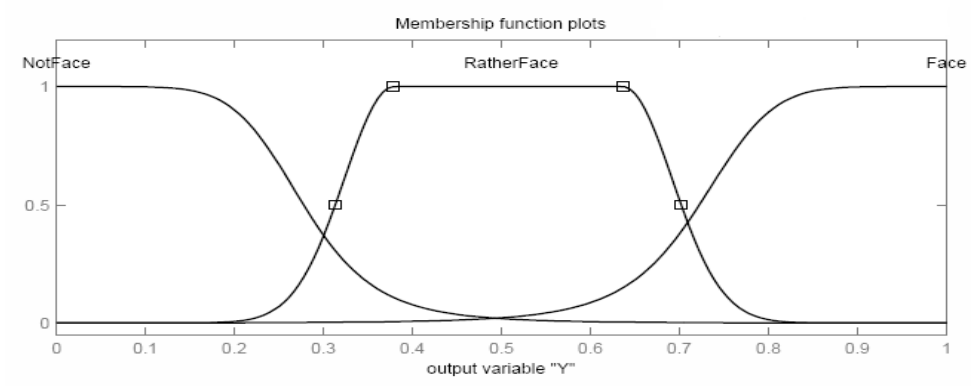

(d)

Figure 3. (a), (b), (c) inputs and (d) output MFs for detecting face candidate fuzzy system
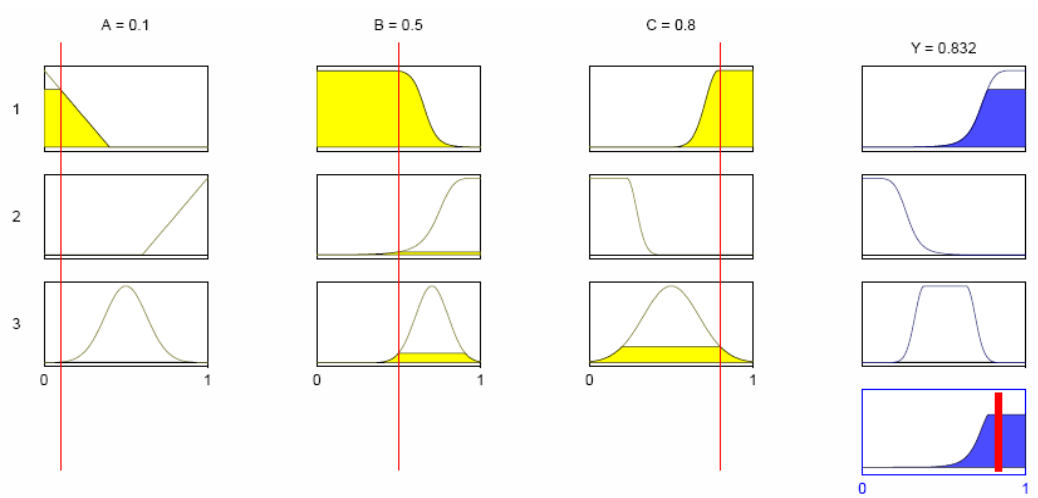

Figure 4. An example of rules composition in detecting face candidate fuzzy system 


\section{CONCLUSION AND DISCUSION}

A new fuzzy rule based method to detect human faces in colour image was proposed and described.

They were three fuzzy systems, respectively to create skinlikelihood gray-scale image, lip-likelihood gray-scale image and select face blobs among skin regions.

Beside colour information, in final decision system, the position and area of lip relative to face area and approximated face shape are used to verify detected faces.

In experimental results, it can be seen that the algorithm works well for frontal faces. The failure of system is about faces in which the lip is not completely observable, such as profile faces. Table I shows the results.

Altering system to identify exact shape of lip in face and detecting profile faces and faces in different pose, are the next program.

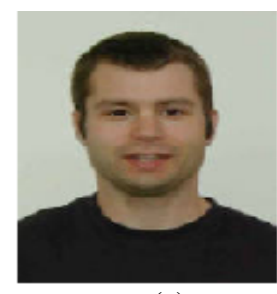

(a)

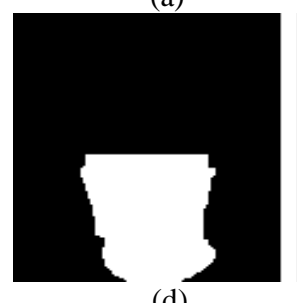

(d)

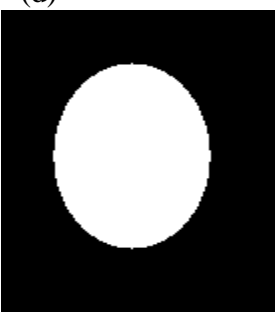

(g)

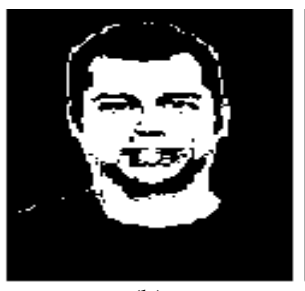

(b)

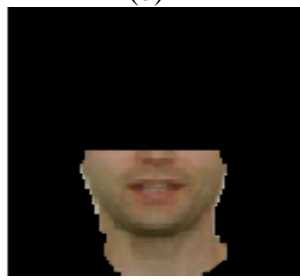

(e)

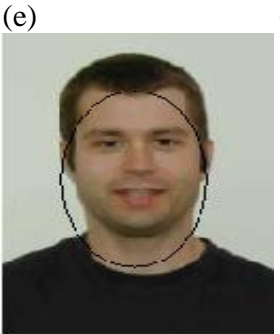

(h)

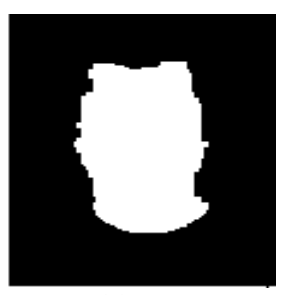

(c)

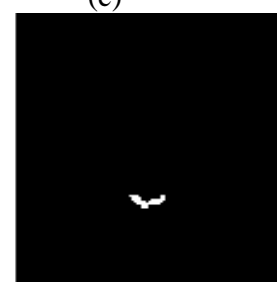

(f)
Figure 5. (a) main image, (b) skin region, (c) skin region after morphological processing, (d) half region, (e) half region transformed to normalised RGB colour space, (f) lip detected area, $(\mathrm{g})$ approximated face shape, $(\mathrm{h})$ detected face in image.

\section{REFRENCES}

[1] M.Yang, D.J.Kriegman, N.Ahuja, "Detecting Face in Image: A Survey", IEEE Transaction on Pattern Analysis and Machine Intelligence, Vol.24, No.1, January 2002.

[2] P.Kakumanu, S.Makrogiannis, N.Bourbaki "A Survey of Skin-Colour Modeling and Detection methods", Pattern Recognition, pp. 1106-1122, 2007.
[3] N.Eveno, A.Caplier, Coulon,"A New Transformation For Lips Segmentation”, Multimedia Signal Processing, 2001 IEEE Fourth Workshop, pp.3-8, Oct.2001.

[4] J.A.Dargham, A.Chekima, "Lips Detection in the Normalised RGB Colour Scheme", Information and Communication Technologies, 2006.ICTTA '06.2nd, Vol.1, pp.1546-1551, April 2006.

[5] J-C.Terrillon, M.David, S.Akamatsu, "Detection of Human Faces in Complex Scene Image by Use of a Skin Colour Model and of Invariant Fourier-mellin Moments", Pattern Recognition, 1998. Proceedings. Fourteenth International Conference, Vol.2, pp.1350-1355, Aug. 1998.

[6] Rein-Lein Hsu, M.Abdel-Mottaleb, A.K.Jain, "Face Detection in Colour Image", IEEE Transaction on Pattern Analysis and Machine Intelligence, Vol.24, pp.696-706, May 2002.

[7] V.Vezhnevets, V.Sazonov, A.Andreeva, "A Survey on Pixel-Based Skin Colour Detection Techniques", Proc.Graphicon-2003, pp.85-92, Moscow, Russia, Sep. 2003.

[8] V.Vezhnevets, A.Andreeva, "A comparative Assessment of Pixel-based Shin Detection Methods"

[9] T.Caetano, S.Olabarriaga, D.Barone, "Performance Evaluation of Single and Multiple-Gaussian Models for Skin Colour Modeling", SIBGRAPI'02: Proceedings of the 15th Brazilian Symposium on Computer Graphics and Image Processing, pp.275-282, 2002.

[10] J.C.Terrillon, M.N.Shirazi，H.Fukamachi， S.Akamatsu, "Comparative Performance of Different Skin Chrominance Models and Chrominance Spaces for Automatic Detection of human Faces in Colour Images", Proc. Of the International Conference on Face and Gesture Recognition, pp.54-62, 2000.

[11] J.Y.Lee, S.I.Yoo, “An Elliptical Boundary model for Skin Colour Detection", Proc. Of the 2002 International Conference on Imaging Science, Systems and Technology, 2002.

[12] K.Xiao, L.Danghui, S.Lansun, "Segmentation of Skin Colour Region Based on Fuzzy Cluster", Proc. Of 2004 International Symposium on Intelligent Multimedia, Video and Speech Processing, Hong Kong, Oct.2004.

[13] S.N.Sivanandum, S.Sumathi, S.N.Deepa, "Introduction to Fuzzy logic using MATLAB", springer-verlag Berlin Heidelberg, 2007.

[14] J.Yen, R.Langary, "Fuzzy Logic”, prentice hall, 1998.

[15] R.Davies, "Machine Vision”, Morgan Kaufman, 2004.

[16] S. Mousavi, P. Moallem, M. Kavosh, A Novel Fuzzy Rule Based Algorithm to Detect Faces with Various Pose, $17^{\text {th }}$ Iranian conference on Electrical engineering, Tehran, Iran, 2009 